\title{
Hydrodenitrogenation Kinetics of Diesel Oil and Catalyst Stacking Simulation
}

\author{
Hongbo Jiang ${ }^{1}$, Xinge Sun ${ }^{1}$, hailong $l^{2}$, Wenbin Chen $^{3}$, Kang Qin ${ }^{3}$, Mingfeng $\operatorname{Li}^{4}$, and \\ Hong $\mathrm{Nie}^{4}$ \\ ${ }^{1}$ Research Institute of Petroleum Processing \\ ${ }^{2}$ East China University of Science and Technology \\ ${ }^{3}$ Sinopec Research Institute of Petroleum Processing \\ ${ }^{4}$ Research Institute of Petroleum Processing, SINOPEC, 18 Xue Yuan Road
}

July 23, 2020

\begin{abstract}
The kinetics of hydrodenitrogenation (HDN) was studied in an isothermally high-throughput reactor over three kinds of catalysts (CoMo, NiMo, NiMoW) to produce ultra-low sulfur diesel. The influences of reaction temperature, reaction pressure, volume ratio of $\mathrm{H} 2$ to oil and space time were systematically investigated to obtain kinetic parameters. By analyzing the reaction mechanism, two-lump kinetic model considering the influence of self-inhibition was proposed for the HDN reaction of diesel oil, and Levenberg-Marquard optimization method was used to estimate the model parameters. The model could well predict the evolution of nitrogen-containing compounds concentration along the axial length of reactor. Based on the two-lump kinetic model, the simulation on the HDN activity of various catalyst stacking schemes is close to the experimental data, which proves the model is applicable for catalyst stacking system. And the concentration of nitrogen-containing compounds was predicted for the catalyst gradation model of different loading sequences.
\end{abstract}

\section{Hosted file}

full.docx available at https://authorea.com/users/345558/articles/471780hydrodenitrogenation-kinetics-of-diesel-oil-and-catalyst-stacking-simulation 